Military Technical College Kobry El-Kobbah, Cairo, Egypt

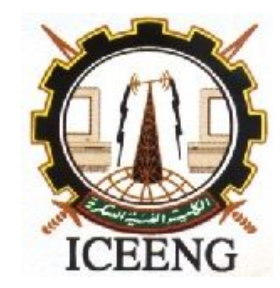

$9^{\text {th }}$ International Conference on Electrical Engineering ICEENG 2014

\title{
A new Optimal Power Flow Algorithms using Particle Swarm Optimization by Matlab. Program
}

\author{
By \\ M.Sherif M.Fahmy *
}

\section{Abstract:}

This paper study, Particle Swarm Optimization for the solution of the optimal power flow (OPF) is studied. Traditionally, classical optimization methods were used to effectively solve OPF. But more recently due to incorporation of Flexible A.C. Transmission System (FACTS) devices and deregulation of a power sector, the traditional concepts and practices of power systems are superimposed by an economic market management. So OPF have become complex. (FACTS) family using power electronics to control power flow and improve transient stability on power grids [1]. In recent years, Artificial Intelligence methods (GA etc) have emerged which can solve highly complex OPF problems. 30-bus system has been studied to show the effectiveness of the algorithm.

\section{Keywords:}

Particle Swarm Optimization (PSO), Flexible A.C. Transmission System (FACTS), Optimal Power Flow (OPF) 
* Egyptian Armed Forces

\section{Introduction:}

The scope of this paper is focused on OPF the main objective is to minimize the fuel cost of meeting the load demand for the power system while satisfying all the security constraints and minimize active power loss. Since OPF is a non-linear problem, decouple of the control parameter of the FACTS device is a highly nonlinear problem so that particle swarm optimization is used as a methodology to solve. In this context, more control facilities may complicate the system operation. As control facilities influence each other, a good coordination is required in order to bring all devices to work together, without interfering with each other. It has also been noted that the OPF problem with series compensation may be a non-convex and non-linear problem, which will lead the conventional optimization method stuck into local minimum. Genetic algorithms and Particle Swarm Optimization offer a new and powerful approach to these optimization problems made possible by the increasing availability of high performance computers. These algorithms have recently found extensive applications in solving global optimization searching problems when the closed-form optimization technique cannot be applied.

\section{$\underline{2 . \ldots \ldots \ldots . . .}$}

The scope of this paper is focused on OPF [2] the main objective is to minimize the fuel cost of meeting the load demand for the power system while satisfying all the security constraints and minimize active power loss. Since OPF is a non-linear problem, decouple of the control parameter of the FACTS device $[1,2,3]$ is a highly nonlinear problem so that particle swarm optimization is used as a methodology to solve. In this context, more control facilities may complicate the system operation. As control facilities influence each other, a good coordination is required in order to bring all devices to work together, without interfering with each other. It has also been noted that the OPF problem with series compensation may be a non-convex and non-linear problem, which will lead the conventional optimization method stuck into local minimum. Genetic algorithms and Particle Swarm Optimization [4] offer a new and powerful approach to these optimization problems made possible by the increasing availability of high performance computers. These algorithms have recently found extensive applications in solving global optimization searching problems when the closedform optimization technique cannot be applied. Genetic algorithms are parallel and global search techniques that emulate natural genetic operators.

The GA is more likely to converge toward the global solution because it, simultaneously, evaluates many points in the parameter space. The method is not sensitive to the starting

Points and capable to determining the global optimum solution to the OPF for range of constraints and objective functions. In this paper a simple genetic algorithm is applied to the problem of optimal power flow. To accelerate the processes of GAOPF, the controllable variables are decomposed to active constraints that effect directly the cost function are included in the Genetic algorithms process and passive constraints which are updating using a conventional load flow program. 


\section{PROBLEM FORMULATION}

The economic dispatch problem [5] is to simultaneously minimize the overall fuel cost rate and meet the load demand of a power system. The power system model consists of $n$ generating units already connected to the system. The economic dispatch problem can be expressed as the most commonly used objective in the OPF problem $[7,8]$ formulation is the minimization of the total cost of real power generation. The individual costs of each generating unit are assumed to be function, only of active power generation and are represented by quadratic curves of second order. The objective function for the entire power system can then be written as the sum of the quadratic cost model at each generator.

$\operatorname{Min} \sum_{i=1}^{n} F i(P i)$

$F_{i} P_{i}=a_{i}+b_{i} P_{i}+c_{i} P_{\mathrm{i}}^{2}$

Where $a_{i}, b_{i}$ and $c_{i}$ are the cost coefficients of $\mathrm{i}$-th generator and $\mathrm{n}$ is the number of generators committed to the operating system. $\mathrm{P}_{\mathrm{i}}$ is the power output of the $\mathrm{i}$-th generator. The economic dispatch problem subjects to the following constraints

$P_{i(\min )} \leq P_{i} \leq P_{i(\max )} \quad$ for $i=1, \ldots \ldots n$

$\sum_{\mathrm{i}=1} P_{i}-P_{D}-P_{L}=0$

Where $P_{L}=\left[P_{1}, P_{2}, \ldots \ldots . P_{n}\right]\left[\begin{array}{ccc}B_{11} & \cdots & B_{1 n} \\ \vdots & \ddots & \vdots \\ B_{1 n} & \cdots & B_{n n}\end{array}\right]\left|\begin{array}{c}P_{1} \\ \dot{P_{n}}\end{array}\right|+\left[P_{1}, P_{2}, \ldots \ldots . . P_{n}\right]\left[\begin{array}{c}\frac{B 01}{2} \\ \frac{B 0 n}{2}\end{array}\right]+\mathrm{B}_{00}$

Where $P_{i}(\min )$ and $P_{i}(\max )$ are the minimum and maximum generating limits respectively for the plant $i . \mathrm{P}_{\mathrm{D}}$ is the load demand and $\mathrm{P}_{\mathrm{L}}$ represents the transmission losses. $B_{i i}$ and $B_{o i}$ are the loss coefficients.

\section{PARTICLE SWARM OPTIMIZATION}

Particle swarm optimization (PSO) is a population based stochastic optimization technique developed by Dr.

Kennedy and Dr. Eberhart in 1995, inspired by social behavior of bird flocking or fish schooling.PSO shares many similarities with evolutionary computation techniques such as Genetic Algorithms (GA). The system is initialized with a population of random feasible solutions and searches for optima by updating generations.

However, unlike GA,PSO has no evolution operators such as crossover and mutation. PSO algorithm has also been demonstrated to perform well on genetic algorithm test function In PSO, the potential solutions, called particles, fly through the problem space by following the current optimum particles .In a PSO algorithm, particles change their positions by flying around in a

multidimensional search space until a relatively unchanged position has been encountered, or until computational limitations are exceeded. In social science context, a PSO system combines a social-only model and a cognition only model. The social-only component suggests that individuals ignore their own 
experience and fine-tune their behavior according to the successful beliefs of the individual in the neighborhood.

\section{DESCRIPTION OF PSO}

In 1995, Kennedy and Eberhart [5,8] first introduced the PSO method, motivated with the social behavior of organisms such as fish schooling and bird flocking. PSO, as an optimization tool, provides a population based search procedure in which individuals called particles change their positions (states) with time. In a PSO system, particles fly around in a multidimensional search space. During flight, each particle adjusts its position according to its own experience, and the experience of neighboring particles, making use of the best position encountered by itself and its neighbors. The swarm direction of a particle is defined by the set of particles neighboring the particle and its history experience.

Let $\mathrm{x}$ and $\mathrm{v}$ denote a particle coordinates (position) and its corresponding flight speed (velocity) in a search space, respectively. Therefore, the $\mathrm{i}^{\text {th }}$ particle is represented as $\mathrm{x}_{\mathrm{i}}=\left(\mathrm{x}_{\mathrm{i} 1}+\mathrm{x}_{\mathrm{i} 2} \ldots \ldots . \mathrm{x}_{\mathrm{id}}\right)$ in the $\mathrm{d}$ dimensional space. The best previous position of the $i^{\text {th }}$ particle is recorded and represented as

Pbest $_{\mathrm{i}}=\left(\right.$ pbest $_{\mathrm{i}}$, pbest $\mathrm{p}_{\mathrm{i} 2} \ldots .$. ..pbest $\left.\mathrm{p}_{\mathrm{id}}\right)$. The index of the best particle among all the particles in the group is represented by gbestd. the rate of the velocity for particle $i$ is represented as $v_{i}=\left(v_{i 1}, v_{i 2}, \ldots, v_{i d}\right)$. The modified velocity and position of each particle can be calculated using the current velocity in distance from pbestid to gbestd.

$\mathrm{V}(\mathrm{k}, \mathrm{j}, \mathrm{i}+1)=\mathrm{w}^{*} \mathrm{~V}(\mathrm{k}, \mathrm{j}, \mathrm{i})+\mathrm{C}_{1} * \operatorname{rand}^{*}(\operatorname{pbestx}(\mathrm{j}, \mathrm{k})-\mathrm{x}(\mathrm{k}, \mathrm{j}, \mathrm{i}))+\mathrm{C}_{2} * \operatorname{rand} *(\operatorname{gbestx}(\mathrm{k})-\mathrm{x}(\mathrm{k}, \mathrm{j}, \mathrm{i}))$

Where, $\mathrm{i}$ is the iteration number

$\mathrm{j}$ is the particle number

$\mathrm{k}$ is the $\mathrm{k}^{\text {th }}$ control variable

$\mathrm{w}$ is the inertia weighting factor

$\mathrm{c}_{1}, \mathrm{c}_{2}$ are acceleration constant

rand () is a uniform random value in the range of $[0,1]$

$\mathrm{V}(\mathrm{k}, \mathrm{j}, \mathrm{i})$ is the velocity of particle $\mathrm{j}$ at iteration $\mathrm{i}$

$\mathrm{x}(\mathrm{k}, \mathrm{j}, \mathrm{i})$ is the current position of particle $\mathrm{j}$ at iteration

$v_{d}{ }^{\min } \leq v_{i d}{ }^{(t)} \leq v_{d}{ }^{\max }$

$x_{i}^{(t)}$ is the current position of particle $i$ at iteration $t$.

in the above procedures, the parameter $\mathrm{v}^{\max }$ determines the resolution, or fitness, with which regions are to be searched between the present position and the target position. If $v^{\max }$ is too high, particles might fly past good solutions. If $\mathrm{v}^{\max }$ is too small, particles may not explore sufficiently beyond local solutions. In many experiences with PSO, $v^{\max }$ was often set at 10-20\% of the dynamic range of the variable on each dimension. The constants $\mathrm{c} 1$ and $\mathrm{c} 2$ represent the weighing of the stochastic acceleration terms that pull each particle toward the pbest and gbest positions. Low values allow particles to roam far from the target regions before being tugged back. On the other hand, high values result in abrupt movement towards, or past, target regions. Hence the acceleration constants $\mathrm{c} 1$ and $\mathrm{c} 2$ were often set to be 2.0 according to past experiences.

Suitable selection of inertia weight $\mathrm{w}$ provides a balance between global and local explorations, thus requiring less iteration on average to find a sufficiently optimal solution. As originally developed, w often decreases linearly from about 0.9 to 0.4 during a run. In general, the inertia weight $\mathrm{w}$ is set according to the following 
equation:

$\mathrm{w}=\mathrm{w}_{\max }-\left(\mathrm{w}_{\max }-w_{\min }\right) /$ iter $r_{\max } \mathrm{x}$ iter

Where: $\mathrm{w}_{\max }$ is the initial weight,

$\mathrm{w}_{\min }$ is the final weight,

iter is the current iteration number,

and iter $_{\max }$ is the maximum iteration number.

\section{GENETIC ALGORITHM}

Genetic algorithms [6] are search algorithms based on the process of biological evolution. In genetic algorithms, the mechanics of natural selection and genetics are emulated artificially. The search for a global optimum to an optimization problem is conducted by moving from an old population of individuals to a new population using genetics-like operators. Each individual represents a candidate to the optimization solution. An individual is modeled as a fixed length string of symbols, usually taken from the binary alphabet. An evaluation function, called fitness function, assigns a fitness value to each individual within the population. This fitness value is measure for the quality of an individual. The basic optimization procedure involves nothing more than processing highly fit individuals in order to produce better individuals as the search progresses. A typical genetic algorithm cycle involves four major processes of fitness evaluation, selection, recombination and creation of a new population. Although the binary representation is usually applied to power optimization problems, in this paper, we use the real valued representation scheme for solution. The use of real valued representation in the GA is claimed by Wright to offer a number of advantages in numerical function optimization over binary encoding. Efficiency of the GA is increased as there is no need to convert chromosomes to the binary type; less memory is required as efficient floating-point internal computer representations can be used directly; there is no loss in precision by discretisation to binary or other values; and there is greater freedom to use different genetic operators. For the real valued representation, the $k$-th chromosome $\mathrm{C}_{\mathrm{k}}$ can be defined as follows:

$C_{k}=\left[P_{k l}, P_{k 2}, \ldots, P_{k l}\right] \quad k=1,2, \ldots$, popsize

Where popsize means population size and Pki is the generation power of the

$i$-th unit at $k$-th chromosome. Reproduction involves creation of new offspring from the mating of two selected parents or mating pairs. It is thought that the crossover operator is mainly responsible for the global search property of the GA. We used an arithmetic cross over operator that defines a linear combination of two chromosomes. Two chromosomes, selected randomly forcross over, $\mathrm{C}_{\mathrm{i}}{ }^{\text {gen }}$ and $\mathrm{C}_{\mathrm{j}}{ }^{\text {gen }}$ may produce two offspring, $\mathrm{C}_{\mathrm{i}}{ }^{\text {gen+1 }}$ and $\mathrm{C}_{\mathrm{j}}{ }^{\text {gen+1 }}$ may produce two offspring, $\mathrm{C}_{\mathrm{i}}{ }^{\text {gen+1 }}$ and $\mathbf{C}_{\mathrm{j}}{ }^{\text {gen+1 }}$, which is a linear combination of theirparents i.e., $C_{i}{ }^{\text {gen+1 }}=a \cdot C_{i}^{\text {gen }}+(1-a) C_{j}^{\text {gen }}$ $\mathrm{C}_{\mathrm{j}}^{\text {gen+1 }}=(1-\mathrm{a}) \cdot \mathrm{C}_{\mathrm{i}}{ }^{\text {gen }}+\mathrm{a} \cdot \mathrm{C}_{\mathrm{j}}^{\text {gen }}$

Where $a$ is a random number in range of $[0,1]$. The mutation operator is used to inject new genetic materialinto the population and it is applied to each new structureindividually. A given mutation involves randomly altering each gene with a small probability. We generate a random real value which makes a random change in the $m$-th element selected randomly of the chromosome. The objective function [9] is used to provide a measure of how individuals have performed in the problem domain. In the case of a minimization problem, the fit individuals will have the lowest value of the associated objective function.

The fitness function is normally used to transform the objective function value into a measure of relative fitness. The fitness function is defined as :Fit $(x)=g(f(x))$ where $f(x)$ is the objective function, $g$ transforms the value of the objective function to nonnegative number. An elitist which GA search is used guarantees that the best solution so far obtained in the 
search is retained and used in the following generation, $\mathrm{n}$ and thereby ensuring no good solution already found can be lost in search process

\section{APPLICATION STUDY}

This work proposes an application of genetic algorithm and Particle Swarm Optimization to solve the Economic Dispatch problems. In this work transmission losses are included by calculating the B coefficients of transmission losses. The results are taken on 30bus system (fig-1) to test the effectiveness of the proposed method. The system consists of 41 lines and 6 generators, bus one is taken as reference bus, others are taken as load buses. The initial angle at respective buses is assumed as zero degree.

Fig-1: 30-bus power system network Generator Operating Costs in $\$ / \mathrm{h}$,

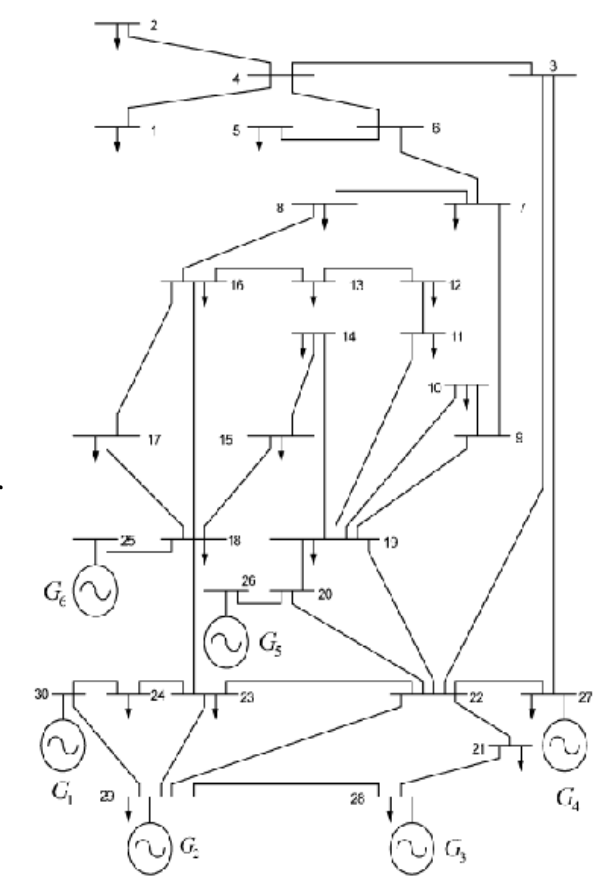

Fig. 1 :30-bus power sys. network 


\begin{tabular}{|c|c|}
\hline Objective Function & Objective Value \\
\hline Active Power Loss & 2.8776 \\
\hline Reactive Power Reserve & 0.3934 \\
\hline Generation Fuel Cost (\$/hour) & 967.13 \\
\hline
\end{tabular}

IEEE 30-Bus System Individual Objective Functions

After Applying Optimization Technique with FACTS

$\begin{array}{lc}\text { Objective Function } & \text { Objective Value } \\ - \text { Active Power Loss } & \text { (MW) } \\ \text { Reactive Power Reserve } & 0.7475 \\ \text { Generation Fuel Cost (\$/hour) } & 966.35\end{array}$

\section{$\underline{\text { 1-Active power loss minimization }}$}

\begin{tabular}{|l|c|}
\hline Without FACTS & $2.8776-0.3934-967.13$ \\
\hline $\begin{array}{l}\text { With Thyristor Control } \\
\text { Series Capacitor TCSC }\end{array}$ & $2.7905-0.4186-966.920$ \\
\hline $\begin{array}{l}\text { With State Var Compensator } \\
\text { SVC }\end{array}$ & $2.8310-0.3631-967.02$ \\
\hline With Both TCSC and SVC & $2.7385-0.3500-966.80$ \\
\hline
\end{tabular}

\section{$\underline{\text { 2-Reactive power Reverse Maximization }}$}

\begin{tabular}{|l|c|}
\hline Without FACTS & $3.7475-0.0932-966.35$ \\
\hline $\begin{array}{l}\text { With Thyristor Control } \\
\text { Series Capacitor TCSC }\end{array}$ & $5.8522-0.0887-867.55$ \\
\hline $\begin{array}{l}\text { With State Var Compensator } \\
\text { SVC }\end{array}$ & $5.4508-0.0440-870.19$ \\
\hline With Both TCSC and SVC & $5.2224-0.0384-894.38$ \\
\hline
\end{tabular}

\section{$\underline{\text { 3-Generation Fuel Cost minimization }}$}

\begin{tabular}{|l|l|}
\hline Without FACTS & $8.6473-0.5942-799.21$ \\
\hline $\begin{array}{l}\text { With Thyristor Control } \\
\text { Series Capacitor TCSC }\end{array}$ & $8.5473-0.6333-798.75$ \\
\hline $\begin{array}{l}\text { With State Var Compensator } \\
\text { SVC }\end{array}$ & $8.6868-0.5685-799.04$ \\
\hline With Both TCSC and SVC & $8.5143-0.4175-798.66$ \\
\hline
\end{tabular}

It is clear that the result for 3 cases is the best for forth case which is the best and the capacitors make help to reduce the losses and all researches told us that if we make $1 \%$ low in the cost system for $1 \mathrm{MW}$ equal save 1 million $\$$ 
Proceedings of the $\mathbf{9}^{\text {th }}$ ICEENG Conference, 27-29 May, 2014

And what about if the system $30 \mathrm{MW}$

\begin{tabular}{|c|c|}
\hline Control Variable & Optimal Value \\
\hline $\mathrm{QC}(10)$ & 0.0450 \\
\hline $\mathrm{QC}(12)$ & 0.0300 \\
\hline $\mathrm{QC}(15)$ & 0.0150 \\
\hline $\mathrm{QC}(17)$ & 0.0350 \\
\hline $\mathrm{QC}(20)$ & 0.0150 \\
\hline $\mathrm{QC}(21)$ & 0.0400 \\
\hline $\mathrm{QC}(23)$ & 0.0350 \\
\hline $\mathrm{QC}(24)$ & 0.0400 \\
\hline $\mathrm{QC}(29)$ & 0.0150 \\
\hline TCL (11) & 0.9875 \\
\hline TCL (12) & 0.9875 \\
\hline TCL (15) & 0.9875 \\
\hline TCL (36) & 0.9875 \\
\hline $\mathrm{VB}(1)$ & 1.1000 \\
\hline $\mathrm{VB}(2)$ & 1.1000 \\
\hline $\operatorname{VB}(3)$ & 1.0821 \\
\hline $\mathrm{VB}(4)$ & 1.0886 \\
\hline $\mathrm{VB}(5)$ & 1.1000 \\
\hline $\operatorname{VB}(6)$ & 1.1000 \\
\hline$P G(2)$ & 0.8000 \\
\hline PG ( 3) & 0.5000 \\
\hline$P G(4)$ & 0.3500 \\
\hline$P G(5)$ & 0.3000 \\
\hline$P G(6)$ & 0.4000 \\
\hline $\operatorname{SVC}(7)$ & 0.0150 \\
\hline $\operatorname{SVC}(8)$ & 0.0050 \\
\hline $\operatorname{SVC}(9)$ & 0.0050 \\
\hline $\operatorname{SVC}(11)$ & 0.0200 \\
\hline SVC (13) & -0.0050 \\
\hline SVC (14) & 0.0250 \\
\hline $\operatorname{SVC}(16)$ & 0.0350 \\
\hline $\operatorname{SVC}(18)$ & 0.0200 \\
\hline SVC (19) & 0.0100 \\
\hline $\operatorname{SVC}(22)$ & 0.0200 \\
\hline SVC (25) & 0.0100 \\
\hline $\operatorname{SVC}(26)$ & 0.0250 \\
\hline $\operatorname{SVC}(27)$ & -0.0250 \\
\hline SVC (28) & 0.0150 \\
\hline $\operatorname{SVC}(30)$ & 0.0150 \\
\hline
\end{tabular}


Table A.2: IEEE 30-Bus System Bus Data

\begin{tabular}{ccccccccc}
\hline $\begin{array}{c}\text { Bus } \\
\text { No. }\end{array}$ & $\begin{array}{c}\mathbf{V} \\
(\mathbf{p u})\end{array}$ & $\begin{array}{c}\mathbf{P}_{\mathbf{G}} \\
(\mathbf{p u})\end{array}$ & $\begin{array}{c}\mathbf{P}_{\mathbf{D}} \\
(\mathbf{p u})\end{array}$ & $\begin{array}{c}\mathbf{Q}_{\mathbf{D}} \\
(\mathbf{p u})\end{array}$ & $\begin{array}{c}\mathbf{Q}_{\text {gmin }} \\
(\mathbf{p u})\end{array}$ & $\begin{array}{c}\mathbf{Q}_{\text {gmax }} \\
(\mathbf{p u})\end{array}$ & $\begin{array}{c}\mathbf{P}_{\text {gmin }} \\
(\mathbf{M W})\end{array}$ & $\begin{array}{c}\mathbf{P}_{\text {gmax }} \\
(\mathbf{M W})\end{array}$ \\
\hline 1 & 1.0500 & 0.00 & 0.0000 & 0.0000 & -0.200 & 2.00 & 0.500 & 2.000 \\
2 & 1.0382 & 48.84 & 0.2170 & 0.1270 & -0.200 & 1.00 & 0.200 & 0.800 \\
3 & 1.0114 & 21.51 & 0.9420 & 0.1900 & -0.150 & 0.80 & 0.150 & 0.500 \\
4 & 1.0194 & 22.15 & 0.3000 & 0.3000 & -0.150 & 0.60 & 0.100 & 0.350 \\
5 & 1.0912 & 12.14 & 0.0000 & 0.0000 & -0.100 & 0.50 & 0.100 & 0.300 \\
6 & 1.0913 & 12.00 & 0.0000 & 0.0000 & -0.150 & 0.60 & 0.120 & 0.400 \\
7 & 1.0000 & 0.00 & 0.2280 & 0.1090 & 0.0 & 0.0 & 0.0 & 0.0 \\
8 & 1.0000 & 0.00 & 0.0760 & 0.0160 & 0.0 & 0.0 & 0.0 & 0.0 \\
9 & 1.0000 & 0.00 & 0.0000 & 0.0000 & 0.0 & 0.0 & 0.0 & 0.0 \\
10 & 1.0000 & 0.00 & 0.0580 & 0.0200 & 0.0 & 0.05 & 0.0 & 0.0 \\
11 & 1.0000 & 0.00 & 0.0240 & 0.0120 & 0.0 & 0.0 & 0.0 & 0.0 \\
12 & 1.0000 & 0.00 & 0.1120 & 0.0750 & 0.0 & 0.05 & 0.0 & 0.0 \\
13 & 1.0000 & 0.00 & 0.0000 & 0.0000 & 0.0 & 0.0 & 0.0 & 0.0 \\
14 & 1.0000 & 0.00 & 0.0620 & 0.0160 & 0.0 & 0.0 & 0.0 & 0.0 \\
15 & 1.0000 & 0.00 & 0.0820 & 0.0250 & 0.0 & 0.05 & 0.0 & 0.0 \\
16 & 1.0000 & 0.00 & 0.0350 & 0.0180 & 0.0 & 0.0 & 0.0 & 0.0 \\
17 & 1.0000 & 0.00 & 0.0900 & 0.0580 & 0.0 & 0.05 & 0.0 & 0.0 \\
18 & 1.0000 & 0.00 & 0.0320 & 0.0090 & 0.0 & 0.0 & 0.0 & 0.0 \\
19 & 1.0000 & 0.00 & 0.0950 & 0.0340 & 0.0 & 0.0 & 0.0 & 0.0 \\
20 & 1.0000 & 0.00 & 0.0220 & 0.0070 & 0.0 & 0.05 & 0.0 & 0.0 \\
21 & 1.0000 & 0.00 & 0.1750 & 0.1120 & 0.0 & 0.05 & 0.0 & 0.0 \\
22 & 1.0000 & 0.00 & 0.0000 & 0.0000 & 0.0 & 0.0 & 0.0 & 0.0 \\
23 & 1.0000 & 0.00 & 0.0320 & 0.0160 & 0.0 & 0.05 & 0.0 & 0.0 \\
24 & 1.0000 & 0.00 & 0.0870 & 0.0670 & 0.0 & 0.05 & 0.0 & 0.0 \\
25 & 1.0000 & 0.00 & 0.0000 & 0.0000 & 0.0 & 0.0 & 0.0 & 0.0 \\
\hline
\end{tabular}




\begin{tabular}{ccccccccc}
\hline 26 & 1.0000 & 0.00 & 0.0350 & 0.0230 & 0.0 & 0.0 & 0.0 & 0.0 \\
27 & 1.0000 & 0.00 & 0.0000 & 0.0000 & 0.0 & 0.0 & 0.0 & 0.0 \\
28 & 1.0000 & 0.00 & 0.0000 & 0.0000 & 0.0 & 0.0 & 0.0 & 0.0 \\
29 & 1.0000 & 0.00 & 0.0240 & 0.0090 & 0.0 & 0.05 & 0.0 & 0.0 \\
30 & 1.0000 & 0.00 & 0.1060 & 0.0190 & 0.0 & 0.0 & 0.0 & 0.0 \\
\hline
\end{tabular}

\section{CONCLUSION}

The scope of this paper anew method with genetic algorithm and Particle Swarm Optimization is presented to solve the optimal power flow problem of power system. Application of these techniques to Optimal Power Flow has been explored and tested. The simulation results show that this simple algorithm can give a good result using only simple modifications. A case study on IEEE test system shows the potential for application of GA\& PSO to determine optimal dispatch of generation with FACTS devices.

\section{REFERENCES}

[1] G. Breuer, "Flexible AC Transmission Systems: Technology for Future", Proceeding of 20th Annual Electrical/ Electronic Insulation conference. Boston MA. October 7-10 1991.

[2] A.J. Wood and B.F. Wollenberg, Power Generation Operation and Control, John Wiley \& Sons, New York, 1984.

[3] Bouktir T., Belkacemi M., Zehar K., Optimal power flow using modified gradient method, Proceedings ICEL'2000, U.S.T. Oran, Algeria, 2000, p. 436-442.

[4] P.H. Chen and H.C. Chang, "Large-scale economic dispatch approach by genetic algorithm," IEEE Trans. on Power Systems, vol. 10, no. 4,pp. 1919-1926, November 1995.

[5] M. Saravanan, S.Mary Raja Slochanal, P.Venkatesh, J.PrinceStephenAbraham," Application of particle swarms optimization technique for optimal location of FACTS devices considering cost of installation and system loadability,"Electr. Power Systems Research, vol.77, pp276-283, 2007.

[6] D. Walters and G.B. Sheble, "Genetic algorithm solution of economic dispatch with valve point loading," IEEE Trans. on Power Systems, vol 8, no. 3, pp. 1325-1331, 1993.

[7] Lie. TT. And Deng W., "Optimal Flexible AC Transmission Systems (FACTS) Devices Allocation", International Journal of Electrical Power and Energy Systems. Vol 19 No2 1999 pp 125134.

[8] Dommel H.W., Tinney W.F., Optimal Power Flow Solutions, IEEE Transactions on power apparatus and systems, vol. PAS.87, No. 10, 1968, p. 1866-1876.

[9] P.H. Chen and H.C. Chang, "Large-scale economic dispatch approach by genetic algorithm," IEEE Trans. on Power Systems, vol. 10, no. 4,pp. 1919-1926, November 1995.

[10] N. G. Hingorani, "Flexible AC Transmission," IEEE Spectrum, Vol. 30, No. 4, pp. 40-45, Apr. 1993. 